\title{
Apprendre avec le jeu numérique Minecraft.edu dans un dispositif interdisciplinaire en collège
}

Leticia Andlauer, Florence Thiault et Laure Bolka-Tabary

\section{(2) OpenEdition \\ Journals}

Édition électronique

URL : http://journals.openedition.org/sdj/1057

DOI : $10.4000 /$ sdj. 1057

ISSN : 2269-2657

Éditeur

Laboratoire EXPERICE - Centre de Recherche Interuniversitaire Expérience Ressources Culturelles Education

\section{Référence électronique}

Leticia Andlauer, Florence Thiault et Laure Bolka-Tabary, « Apprendre avec le jeu numérique Minecraft.edu dans un dispositif interdisciplinaire en collège », Sciences du jeu [En ligne], 9 | 2018, mis en ligne le 13 juin 2018, consulté le 19 avril 2019. URL : http://journals.openedition.org/sdj/1057 : DOI : $10.4000 /$ sdj. 1057

Ce document a été généré automatiquement le 19 avril 2019

Tous droits réservés 


\section{Apprendre avec le jeu numérique Minecraft.edu dans un dispositif interdisciplinaire en collège}

Leticia Andlauer, Florence Thiault et Laure Bolka-Tabary

\section{Pour une approche extensive du jeu vidéo en contexte éducatif}

1 Le jeu est depuis longtemps utilisé comme support pédagogique. Toutefois, dans un contexte où le lien qu'entretiennent les jeunes envers les outils issus des Technologies de l'Information et de la Communication (TIC) suscite à la fois crainte et intérêt de la part des pouvoirs publics, la mise en place d'un objet comme le jeu vidéo en classe fût et reste très progressive. Dans les années 2000, cette attention ne concerne pas uniquement les jeux vidéo, mais également l'usage des réseaux sociaux, la consultation des contenus, les modes de consommation des images, etc. Tandis que les systèmes éducatifs sont confrontés à un contexte de crise (multiples réformes, dévalorisation de l'image et perte des vocations des enseignants etc.), la place des TIC au sein des pratiques pédagogiques devient l'objet de nouveaux enjeux éducatifs et pédagogiques. Ces dispositifs sont alors vus comme «à la fois un des agents de la crise et un des moyens de la résoudre pour peu qu'on leur donne une dimension pédagogique » (Dumas, 2004). En 2007, Laurent Trémel interroge les liens entre jeux, éducation et socialisation afin d'inciter les chercheurs à se pencher sur le jeu et de ses mécaniques à travers une approche compréhensive des jeux vidéo, recentrée sur leur nature et leurs caractéristiques ludiques (Tremel, 2007).

2 Progressivement, intégrer une dimension pédagogique aux médias et jeux vidéo devient l'enjeu de nouvelles recherches et de nouveaux projets. De cette manière, de nombreux travaux (Brougere, 2002 ; Alvarez, Djaouti, Rampnoux, 2016 ; Lavigne, 2016) interrogent le potentiel éducatif de ces objets jusqu'ici tenus à l'écart de l'école et cherchent à leur apporter de la légitimité. Dans le même temps, les encouragements des instances éducatives envers des formes de pédagogies innovantes vont également permettre aux 
jeux vidéo, et aux nouveaux objets que sont les serious games ou jeux sérieux de trouver une place au sein des salles de classe. Il s'agit aujourd'hui d'observer les évolutions de cette appropriation de la culture vidéoludique par les systèmes éducatifs à la fois du point de vue des acteurs, mais également du côté des élèves et des pratiques qui se déroulent en classe.

Ces derniers plébiscitent en effet ce type d'activité vidéoludique qui les renvoie à leurs propres pratiques culturelles (Octobre, 2004). Outre le fait qu'il séduit les élèves, le jeu numérique offre de nouvelles possibilités pour l'enseignement. La simulation réaliste associée au plaisir du jeu permet une entrée différente dans les apprentissages. Toutefois, l'attitude des élèves face à ce qui est considéré par les instances éducatives comme un jeu mérite une attention particulière et demande à être relativisée. En se référant aux travaux de Jacques Henriot (1989), on peut considérer "l'idée de jeu» comme ancrée dans des contextes - sociaux, historiques et géographiques - selon lesquels cette idée de jeu, et par conséquent l'attitude du joueur, peuvent différer. De plus, l'un des apports de sa pensée fut de considérer qu'il est impossible pour un observateur extérieur de certifier si la personne observée joue effectivement. C'est à cette dernière d'énoncer sa pratique comme relevant du jeu. La situation d'interaction, qui se déroule à l'école dans un dispositif de médiation entre enseignant, objet vidéoludique et apprenant, engage les élèves dans une démarche d'apprentissage et d'expérimentation au pouvoir motivationnel. Cette même situation leur fait accepter un ensemble de règles propres à ce qui est, dans ce contexte, considéré comme jeu et qui structurent leur comportement en classe.

4 Les principales difficultés rencontrées dans la mise en place de jeux sérieux à l'école restent l'entrée dans le jeu ainsi que l'adoption par les élèves d'une attitude de jeu qui se heurte souvent à un contenu trop didactique et pas assez ludique. Alors que l'outil d'apprentissage est présenté comme un jeu, la situation créée en classe est-elle vécue comme ludique par les individus présents? Comment les élèves appréhendent-ils les pratiques du jeu en classe à partir de leurs propres expériences de joueurs ? Il s'agit là de déterminer les modalités de l'appréhension par les élèves d'un jeu vidéo en tant que support d'une pédagogie active et de questionner la nature de ce type d'activité pour l'apprentissage. Notre analyse s'organise autour de deux axes principaux: les pratiques vidéoludiques de la population étudiée dans les activités scolaires et dans le contexte familial et la question des apprentissages informels avec le jeu vidéo. En effet, l'usage, dans un cadre d'apprentissage institutionnel, d'un jeu vidéo pensé initialement pour le divertissement nécessite de s'interroger sur la façon dont sont intégrés les effets éducatifs d'une pratique ludique dans un dispositif didactique.

\section{Observer l'expérience de Minecraft.edu en contexte éducatif}

5 Minecraft (jeu de type bac à sable ${ }^{1}$ ), dont le développement a débuté en 2009, est parfois nommé le «Lego du XXI e siècle » par les médias; ce jeu doit son succès au fait de pouvoir créer un monde fait de blocs, que l'on peut récupérer, transformer et manipuler au gré de l'imagination pour construire des bâtiments et des mondes imaginaires. Il n'y a donc pas de trame narrative dans Minecraft. Les joueurs peuvent sélectionner la difficulté du jeu et choisir leur mode de jeu (seul ou à plusieurs). Ils sont amenés soit à construire ensemble (mode créatif) soit à se battre (mode survie) ${ }^{2}$. Ce jeu vidéo fut utilisé dans de nombreux 
pays comme outil d'éducation pour aborder différentes thématiques: la revue de littérature proposée par Nebel, Schneider et Rey (2016) sur l'utilisation de Minecraft pour l'éducation évoque des sujets variés allant de la géométrie spatiale à l'écologie en passant par les matières plus classiques telles la géographie, la biologie, l'histoire, les arts, etc. Pensée spécifiquement pour être utilisée en contexte éducatif, la version Minecraft.edu ${ }^{3}$ est sortie au cours de l'été 2016. Elle propose notamment une interface plus simple d'utilisation pour les professeurs et se limite au mode créatif pour les élèves. A la fois perçu comme ludique, car bénéficiant de l'image d'un jeu vidéo de par sa version originale, et répondant ainsi aux intérêts du système éducatif pour le numérique et l'utilisation des TIC en classe, Minecraft.edu semble alors être un outil idéal pour mettre en place un scénario d'apprentissage par le jeu.

Durant l'année scolaire 2016-2017, les enseignants d'un collège des Hauts de France ont choisi de recourir à l'usage de Minecraft.edu avec l'ensemble des classes de $5^{\mathrm{e}}$ dans le cadre d'un Enseignement Pratique Interdisciplinaire (EPI). L'objectif est de reconstruire une seigneurie médiévale avec l'abbaye, le château et le village. Les disciplines mobilisées interviennent sur des points complémentaires. L'enseignant d'histoire se charge de l'affectation des bâtiments à construire aux différentes classes puis de la mise en vie de la seigneurie. Les enseignants de mathématiques travaillent sur l'échelle des différents bâtiments et la réalisation des fondations. Quant aux professeurs de technologie et d'arts plastiques, ils supervisent les étapes de la construction ainsi que le choix des matériaux et de décoration.

$7 \quad$ Les utilisations en classe étant limitées au mode créatif, elles divergent assez fortement de l'expérience du jeu des jeunes hors contexte scolaire (qui privilégient le mode survie). Une élève joueuse experte nous décrit le jeu comme suit :

Minecraft, c'est un peu un type de jeu bac à sable. En gros, y a plusieurs types de jeu : y a la survie, y a le créatif, le hardcore, et le ultra hardcore. La survie c'est que tu dois survivre avec une barre de vie et une barre de nourriture. Et tu as plusieurs vies, si tu meurs tu peux respawn [réapparaître]. En hardcore c'est que tu peux regen [régénérer], mais tu peux pas vivre plusieurs fois, tu peux pas respawn. Dès que tu meurs c'est fini. Et l'ultra hardcore c'est que tu peux pas regen et que t'as une seule vie. Et le créatif tu peux faire tout ce que tu veux, tu peux voler, et t'as pas de barres de vie ni de nourriture. Tu peux jamais mourir. (extrait entretien élève)

Cette description des modes de jeu met en avant le fait que le mode créatif ne semble pas présenter de règles ou d'objectifs ludiques. L'élève se trouve face à une liberté qui devra être cadrée dans la situation d'une utilisation en classe. Il est judicieux de s'interroger sur la nature de ce dispositif : puisque Minecraft.edu a été conçu pour un usage pédagogique, et si les règles pour l'élève sont dictées par la situation éducative, alors l'idée de jeu est ici remise en cause. Alors que la revue de littérature évoquée précédemment relève de nombreux travaux consacrés à l'analyse de la mise en place des dispositifs autour de Minecraft (Nebel et al., 2016), cet article entend se recentrer sur la parole des élèves afin de déterminer la nature de leur activité en classe.

Nous avons adopté une méthodologie de recherche basée sur une enquête et des observations directes afin de croiser données qualitatives et quantitatives, informations déclaratives et observées. Cette méthodologie a permis d'obtenir des données factuelles sur le déroulement de l'EPI, sur son appréhension par les enseignants et les élèves, sur la motivation des élèves et les dynamiques de groupe. Des entretiens semi-directifs ont été menés auprès de 16 élèves issus des six classes concernées par l'EPI. Les élèves ont été interrogés par trinôme, à l'exception d'une classe où une seule élève s'est présentée à 
l'entretien. Les entretiens d'une trentaine de minutes ont été réalisés par deux chercheuses, l'une menant l'entretien, l'autre prenant des notes. Les élèves ont été interrogés à la fois sur le jeu Minecraft et son intérêt éducatif, les transferts de compétences entre le jeu et les disciplines scolaires, ainsi que leurs pratiques ludiques et vidéoludiques personnelles. Il s'agissait de déterminer quelles pratiques de loisirs ils assimilent au jeu, dans quels contextes, ainsi que la façon dont elles sont décrites, afin d'éclairer les modalités de construction de leur perception de l'activité menée durant les séances avec Minecraft.edu.

10 Ces entretiens visaient à compléter une première enquête par questionnaire menée auprès de l'ensemble des élèves de $5^{\mathrm{e}}$ (153 répondants). Le choix des élèves interrogés a été fait par l'équipe enseignante, à qui nous avions préalablement demandé de sélectionner des profils d'élèves variés en fonctions de critères tels que le sexe, l'expertise dans le jeu, le niveau scolaire, le comportement et la socialisation dans le groupe. Des entretiens semi-directifs ont également été réalisés avec quatre enseignants des quatre disciplines représentées dans l'EPI. Nous avons demandé aux enseignants de nous raconter la genèse du projet, son déroulement, les liens avec leur discipline, les difficultés rencontrées et leur avis sur cette expérience.

11 Parallèlement à ce travail d'enquête, nous avons effectué des observations de séances d'EPI : une séance de mathématiques, une séance de technologie et deux séances d'arts plastiques. Ces séances se déroulaient en salle informatique. Nous étions installés en fond de classe, dans une posture d'observation non participante, et nous nous déplacions pour observer et photographier les informations disposées par l'enseignant sur un tableau ainsi que les écrans des élèves. Nos observations se sont concentrées sur le déroulement de la séance, les consignes, déplacements et interactions de l'enseignant avec les élèves, les déplacements et interactions des élèves entre eux (dans la classe et au sein des groupes de travail), leur comportement pendant la séance et leur participation effective au travail collectif. Pour clôturer l'enquête, un questionnaire de bilan a porté sur l'évaluation de l'expérience de jeu et les représentations des compétences acquises des élèves (117 répondants).

\section{La place des jeux vidéo dans les loisirs des élèves}

12 Le jeu vidéo constitue un objet incontournable de la culture adolescente. L'enquête PELLEAS (Programme d'étude sur les liens et l'impact des écrans sur l'adolescent scolarisé) $)^{4}$ menée par la Croix-Rouge en 2014 a constaté que $94 \%$ des garçons et $84 \%$ des filles interrogées déclarent jouer à un jeu vidéo de façon hebdomadaire. Lors de notre enquête, les jeux les plus cités par les élèves appartiennent aux genres Sport, ActionAventure et Jeu de tir à la première personne : il s'agit de FIFA Football 17 (2016), GTA 5 (Grand Theft Auto, 2013) et Call of Duty (COD, 2016). S'ils sont cités très souvent, et majoritairement par des garçons, les filles indiquent également aimer ces jeux. Il faut toutefois rappeler que GTA et COD sont déconseillés aux moins de 18 et 16 ans, et que les jeunes interrogés ont de 12 à 13 ans. Néanmoins, parmi l'ensemble des jeux cités, seuls ces trois titres très populaires font consensus auprès des élèves. Beaucoup de jeux ne sont mentionnés qu'une voire deux ou trois fois: ils présentent cependant une grande diversité de catégories et de titres, mais également de supports, avec des jeux sur console, $\mathrm{PC}$, console portable et également tablettes et smartphones. Parmi tous les titres évoqués, et bien qu'il ne soit pas aussi présent que FIFA, GTA et COD, Minecraft se démarque et se 
trouve aussi bien cité par des filles que des garçons, sans doute en lien avec l'expérience scolaire en cours.

Notre enquête par questionnaire auprès des collégiens, nous révèle en effet que deux tiers des élèves jouaient déjà à Minecraft chez eux avant de l'utiliser en cours et que $83 \%$ jouaient également à d'autres jeux. Les réponses nous informent sur leur temps de jeu qui correspond pour presque la moitié d'entre eux à moins d'une heure par jour, 34,5\% déclarant jouer de une à trois heures par jour et $19 \%$ plus de trois heures par jour. Ils jouent le plus souvent sur une console de salon type Playstation, Wii ou Xbox, qui peut être une console familiale, personnelle, parfois héritée du père suite à l'acquisition d'un modèle plus récent. Enfin, l'appétence des filles par rapport aux jeux vidéo semble moindre que celle des garçons, et les résultats du questionnaire confirment ces tendances: davantage de filles déclarent ne pas jouer, tandis que les garçons dans l'ensemble se disent davantage attachés à cette pratique.

Figure 1 : Proportion des joueuses et joueurs de Minecraft avant les séances en EPI

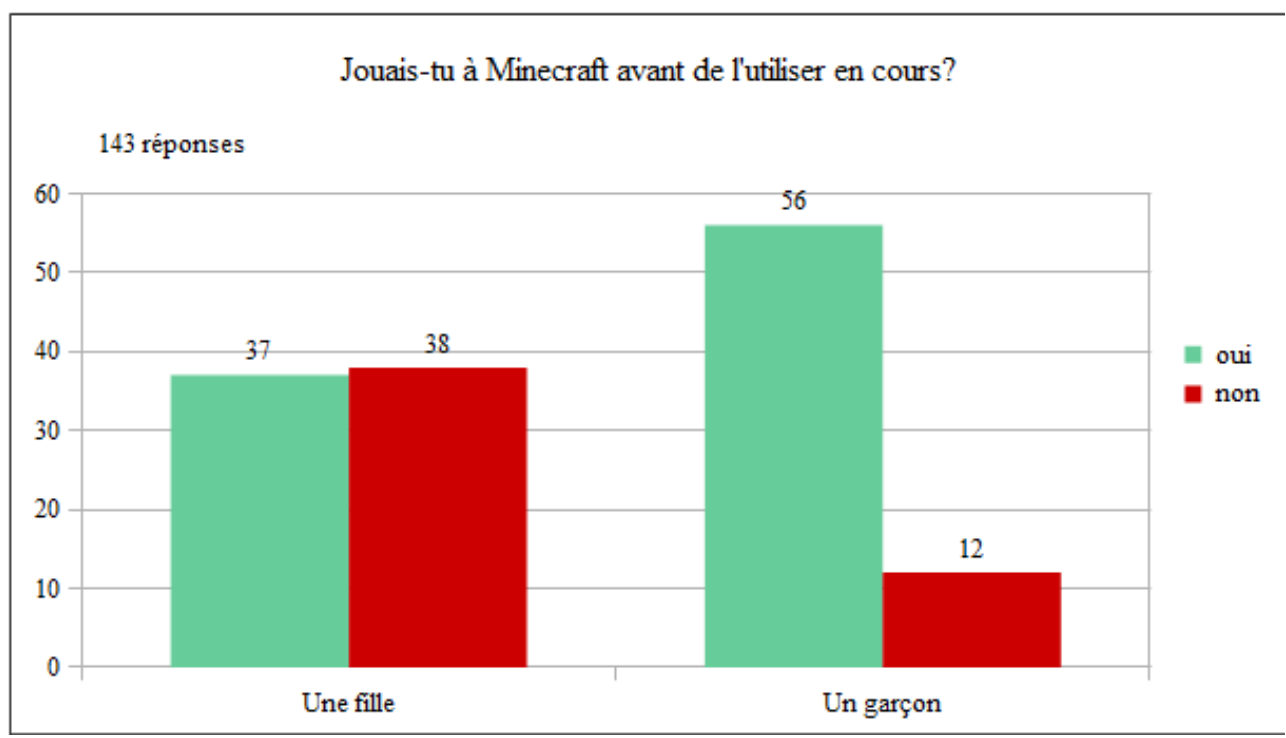

Les collégiens rencontrés en entretiens ont des pratiques variées des jeux vidéo, mais se révèlent majoritairement être des joueurs modérés, pour lesquels le jeu vidéo s'avère une pratique de loisirs parmi d'autres (un seul collégien déclare jouer plus de trois heures par jour). La plupart pratiquent une activité sportive (natation, basket, tennis de table, football, équitation) et/ou musicale, qui leur prend souvent plusieurs heures par semaine. Ils déclarent tous regarder des vidéos sur Youtube, en citant essentiellement des vidéastes comiques et, pour les joueurs les plus assidus, les diffusions en mode continu (stream) de jeux vidéo ${ }^{5}$ (Minecraft ou autres jeux pratiqués à la maison). Ils regardent également des films et séries (plusieurs citent la plateforme Netflix) avec une prédilection pour le genre « horreur». La lecture est citée plus marginalement (mangas, Harry Potter, romans policiers...). Les garçons aiment retrouver leurs amis à l'extérieur pour jouer au football, faire du vélo ou de la trottinette, se balader, les filles apparaissant comme plus sédentaires.

Les pratiques ludiques privées des jeunes conditionnent l'appréhension du jeu en séance pédagogique. Lavigne (2016) différencie deux groupes de joueurs : les gros joueurs (GJ) et les petits joueurs (PJ). Ces deux groupes accordent de l'importance à la dimension sociale 
de l'activité ludique. Les GJ jouent par passion et l'esprit de compétition est un marqueur important d'investissement dans l'activité ludique. Ils constituent un public sensible aux différentes caractéristiques traditionnelles d'un jeu vidéo (scénario, graphismes...). De leur côté, les PJ jouent à des jeux de façon occasionnelle, leur principale motivation étant de s'amuser ou chasser l'ennui.

Ce rapport différencié au jeu vidéo s'est vérifié lors des entretiens où ce dernier est mentionné à plusieurs reprises par des PJ comme une activité non préférentielle, sur laquelle ils se rabattent quand ils n'ont rien de mieux à faire ; les GJ mentionnent quant à eux le jeu vidéo comme étant leur activité préférée, qu'ils pratiqueraient de manière plus intensive si leurs parents ne contrôlaient pas leur temps d'écran. En effet, la plupart des familles ont instauré des règles pour surveiller le temps de jeu telles des limitations horaires : « sur le PC mon père m'a mis un truc : ça commence à $13 \mathrm{~h}$ et ça se termine à $23 \mathrm{~h}$ pendant les week-end. Et pendant l'école c'est de $18 \mathrm{~h}$ à $21 \mathrm{~h}$ ». Les deux catégories de joueurs perçoivent de façon différente le fait de jouer en classe.

Fille 2 (F2) : Les séances Minecraft c'est plus du jeu que des séances normales. [GJ]

Chercheuse (C) : Pour ceux qui jouaient pas à Minecraft, est-ce que ça vous a plu?

Garçon 3 (G3) : Moi j'aime bien.

F3 : Moi aussi j'aime bien, mais je joue pas. En fait je fais que quand je suis en cours :

pas en dehors des cours.

$\mathrm{C}: \mathrm{Tu}$ veux dire que pour toi c'est pas du jeu?

F3 : Pour moi non.

$\mathrm{C}:$ Et pour vous deux, vous estimez jouer en cours ou pas?

G4 : Non.

G3 : Ouais un peu. C'est un peu les deux parce que les profs nous laissent un peu

libres mais il y a certaines limites : on doit respecter ce qu'on doit faire.

Il y a donc plusieurs types d'appréhension de l'expérience des «séances Minecraft ». La pratique du jeu et l'idée de jeu que construisent les élèves réfèrent à des contextes précis, de loisirs partagés ou en solitaire, qui sont cadrés et déjà définis comme tels dans un environnement familial ou amical. Par conséquent, ces appréhensions du jeu diffèrent selon les individus interrogés. Les GJ ou experts ont une idée précise des éléments constitutifs qui devraient composer un jeu : de l'amusement, une activité dite "libre ", et les caractéristiques particulières de la version originale de Minecraft (mode survie, challenges, etc.). Cette représentation peut entrer en conflit avec l'expérience de jeu en séance pédagogique. Les PJ sont à l'inverse plus sensibles aux artifices ludiques des jeux sérieux. Enfin, certains (PJ) déclarent cependant, durant les observations en séance, ne pas s'amuser particulièrement et simplement attendre les consignes à suivre pour l'avancement du projet.

\section{Minecraft.edu, un jeu sérieux?}

18 L'utilisation de Minecraft.edu en classe s'apparente au fait de détourner les mécaniques d'un jeu connu par les élèves pour un objectif autre que le seul jeu. Son usage à des fins pédagogiques a pour aspiration de permettre aux élèves de construire numériquement une seigneurie médiévale par l'entremise d'un dispositif ludo-numérique. Pour Alvarez et Djaouti :

Ce type d'application informatique s'appuie sur le jeu vidéo, tant sur le plan technique que culturel, pour tenter de s'écarter du divertissement. Ce qu'on pourrait traduire en français par «jeu sérieux", ou plutôt «jeu à intention 
utilitaire ", a pour principale vocation d'apprendre, d'informer, d'expérimenter, de s'entraîner tout en jouant. (Alvarez, Djaouti, 2012, p. 9)

19 Le jeu sérieux utilise donc les mécanismes du jeu vidéo pour délivrer un contenu pédagogique selon des objectifs clairement définis assignés par les concepteurs. Contrairement au jeu sérieux, où l'intention sérieuse prime sur la visée ludique, le détournement éducatif du jeu vidéo (Serious Gaming), vise à exploiter des jeux vidéo commerciaux à des fins d'apprentissage. Ce terme comprend toutes les approches de détournement de jeux dédiés au divertissement pour aboutir à des finalités sérieuses non anticipées par ses concepteurs afin d'attribuer au jeu des nouvelles fonctions. A priori, dans ce cas de figure, les enseignants disposent d'une plus grande marge de manœuvre pour élaborer un projet didactique. Le détournement éducatif du jeu vidéo (Serious Gaming) peut s'effectuer de deux manières: par une modification des usages (Serious Diverting) et par une modification de l'artefact qu'est le jeu (Serious Modding) (Alvarez, Djaouti et Rampnoux, 2016). Ainsi, la version éducative de Minecraft offre de multiples fonctionnalités supplémentaires, que ce soit en termes d'administration du serveur (avec des raccourcis commandes pour téléporter les joueurs et une carte pour voir où ils se trouvent) ou en termes de mécanismes de jeu et jouabilité avec des ajouts de modifications (mods) directement intégrés au jeu tel que « Architecture Craft » offrant de nouveau blocs et fonctionnalités plébiscités par les élèves. « Il y a plein de blocs qu'il n'y a pas dans le vrai Minecraft donc c'est sympa parce qu'on pouvait faire des arches, des cercles » (extrait d'un entretien élève).

20 Au vu de ces éléments, il apparaît que l'utilisation de Minecraft telle qu'elle se présente au collège observé relève d'une situation de modification de l'artefact (Serious Modding) visant à faciliter l'usage et l'intégration du jeu vidéo en classe. Cependant, pour les joueurs experts, jouer en contexte éducatif apparaît comme une expérience contradictoire :

Je trouve que Minecraft ici on a un truc à faire et on est obligés de le faire. Alors que Minecraft à la maison j'ai le droit de faire ce que je veux. Je veux faire une survie ${ }^{6}$ je vais aller faire une survie. Alors qu'ici c'est du genre tu construis une maison et après tu vas aider les gens, et tu vas pas dire « je vais faire une survie » : j'ai pas le droit.

21 Lorsqu'on les interroge sur le sentiment de jouer ou de travailler pendant ces séances, ils répondent que « c'est entre les deux » et insistent sur les différences d'attitudes scolaires. «Bah, Minecraft on le prend au sérieux mais on peut quand même rigoler. C'est un jeu. Alors que pendant les vrais cours on doit être sérieux, on doit rester calmes, on peut pas parler ». Le fait de vivre un moment scolaire qui contraint moins le corps en ce qui concerne la posture physique (pouvoir se déplacer) et l'expression (discuter avec ses camarades) génère une adhésion forte pour ce type d'activité : «On préfère les séances Minecraft que les séances normales ». Ces entretiens révèlent ainsi que la définition de l'activité vécue pendant ces «séances Minecraft" reste floue: elle ne répond pas à l'expérience de jeu telle que les élèves la conçoivent à partir de leurs pratiques de loisirs, et ne correspond pas non plus à ce qu'ils qualifient de « vrai cours ». Il semblerait que les élèves désignent l'activité durant les séances pédagogiques comme « jeu » car l'utilisation de Minecraft.edu les renvoie à une expérience vécue dans un contexte différent avec le « vrai » Minecraft. Il est également probable que les professeurs, considérant Minecraft.edu comme un jeu et en tant que médiateurs du dispositif pédagogique mis en place, influencent la perception et la définition de cette activité chez les élèves. 


\section{Mécanisme de jeu et apprentissage informel} (gestion, stratégie, réflexes, rapidité...). Jouer à des jeux vidéo, c'est en effet apprendre une nouvelle littératie et acquérir des compétences liées à la maîtrise des technologies numériques. Ce point est souligné par un élève interviewé : «je jouais pas sur ordi du coup il y avait des modes [dans Minecraft] autres que sur la console. Du coup je savais pas qu'on pouvait faire ça ». L'expérience du jeu Minecraft.edu en collectif favorise chez les apprenants joueurs une amélioration de leurs compétences socio-cognitives et digitales : la prise d'initiative, l'autonomie, la résolution de problème. Jouer en classe développe les interactions et la socialisation pour certains élèves plus faibles. Effectivement, l'utilisation du jeu encourage la coopération entre les joueurs qui doivent partager leurs informations pour résoudre les problèmes rencontrés : «Quand nous on voulait le faire et qu'on voyait quelqu'un y arriver on disait : est-ce que tu peux me laisser faire parce que moi je sais pas faire par exemple ceci, et toi tu sais le faire donc on va aider les autres » (extrait entretien élève). Ainsi cette organisation pédagogique met en place une intelligence collective dont les caractéristiques se fondent sur une décentralisation du savoir et des pouvoirs (de l'enseignant non spécialiste du jeu à l'élève expert) : «Des fois 
les profs ils sont un peu moins doués que nous dans Minecraft du coup on leur demande si ça va et après ils nous demandent même si on pourrait faire ça. Les rôles sont un peu inversées" (entretien élève). L'autonomie des individus est valorisée en tant que créateurs.

La situation est structurée de manière coopérative. Les individus travaillent ensemble vers un but commun. Ils perçoivent qu'ils ne peuvent réaliser leurs objectifs que si les autres membres du groupe atteignent également leur but. Selon Brougère, "vouloir participer, faire avec d'autres est générateur d'apprentissages pour partie conscients et formels, pour partie non intentionnels et informels» (Brougère, 2012, p.12). Cet apprentissage informel est effectué par l'échange entre pairs (entre joueurs du groupe classe ou d'autres classes du projet) : «des élèves d'une autre classe sont venus parce qu'ils avaient trouvé des trucs dans Minecraft, pour nous montrer. C'était pour faire les toits en pente et pas en escalier ». En effet, l'activité de recherche d'informations (recours aux documents pédagogiques fournis par les enseignants : dessins d'époque des éléments constitutifs d'une seigneurie tel un four à pain) est structurante pour organiser le travail. Le fait que plusieurs classes travaillent sur le même projet avec des bâtiments spécifiques à construire (abbaye, château, maisons du village, four et puits, moulins, gibet...) nécessite une interactivité constante entre les joueurs et l'environnement technique dont les modifications sont perçues et contrôlées en temps réel par le suivi sur la carte commune, projetée sur écran.

\section{L'intégration du jeu Minecraft dans la pédagogie}

L'intérêt d'un jeu dépend surtout de la manière dont l'enseignant l'intègre dans le dispositif de formation. La question de l'évaluation des apprentissages est fondamentale. Dans le cadre de la réforme du collège, notre expérimentation portait sur un nouveau dispositif d'enseignement interdisciplinaire. Les élèves rencontrés en entretien ont révélé des difficultés à définir cet enseignement. Seul un élève, fils d'enseignant, a su caractériser cet enseignement comme "un travail interdisciplinaire avec plusieurs matières ». Pour autant, certains d'entre eux réalisent l'intérêt de la complémentarité des disciplines pour élaborer le projet collectif : «En fait on se rend compte que les matières elles sont un peu liées. Parce que faire la technologie c'est plus pour construire des choses tout ça mais pour les construire il faut connaître bien les maths ». Pour la majorité, les séances pédagogiques menées en EPI se sont révélées principalement des temps privilégiés pour réaliser un projet de groupe ( $86 \%$ des réponses au questionnaire bilan) et apprendre autrement (60\%).

Dans le contexte du socle commun de connaissances, de compétences et de culture, l'évaluation par compétences qui prend une autre forme qu'une évaluation sommative classique n'est pas toujours bien appréhendée par les élèves : «C'est des compétences, c'est la nouvelle réforme. C'est plus des notes, c'est comme en maternelle. Rouge c'est mauvais, jaune c'est en cours d'acquisition, vert c'est acquis et vert foncé c'est très bien, expert ». Ils pensent surtout être évalués sur leur comportement et leur participation: " si on est sages, si on cherche sérieusement ». En fait, ils n'ont pas vraiment conscience d'une évaluation en œuvre. En effet, seulement $57 \%$ d'entre eux estiment avoir été évalués lors des séances. Un quart des élèves déclarent ne pas savoir si une évaluation a eu lieu. Quant à $15 \%$ des élèves, ils n'ont pas le sentiment d'avoir été évalués par les enseignants sur ces exercices. Cette difficulté pour identifier les points d'évaluation peut 
s'expliquer par le fait que les élèves ne perçoivent pas ces séances comme des cours et ne leur accordent donc pas une « légitimité » pédagogique.

Figure 2 : Impressions des élèves sur l'évaluation de l'EPI

\section{As-tu été évalué sur ces cours?}
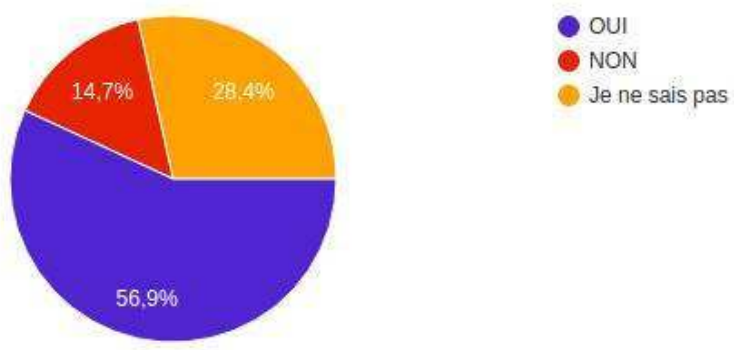

L'action menée dans le jeu se révèle première pour les apprenants. Ainsi, lorsque l'on demande aux élèves ce qu'ils ont appris en histoire, la moitié évoque la phase de défrichage du terrain (activité menée lors de la première séance avec le professeur d'histoire). Néanmoins, une partie des élèves conceptualise effectivement le thème de travail comme « l'architecture au Moyen-âge » et comme possible élément d'évaluation : «si on fait des bâtiments qui ressemblent pas à des bâtiments du Moyen-âge, ça va pas être acquis. Des carreaux, des vitres ça existait pas ». L'étude des échelles de mesure en mathématiques s'avère être plus évidente pour les élèves car une partie de cet enseignement s'est déroulé en classe avant la transposition sur les plans de l'abbaye virtuelle. Ils ont appliqué dans le jeu ce qu'ils avaient préparé au préalable sur des plans papiers : «sur des feuilles à petits carreaux, on avait colorié par exemple, s'il y avait 74 blocs, on coloriait 74 blocs, on faisait la largeur et on a dessiné l'abbaye au sol en fait, sur une feuille de carreaux ». Ces deux exemples montrent bien que le scénario pédagogique reste central comme lors d'un enseignement ordinaire.

Le jeu constitue un dispositif d'apprentissage parmi d'autres. Au-delà des compétences transférables acquises par la pratique collective du jeu, la manipulation de systèmes complexes et l'apprentissage par essais erreurs leur a permis de développer des compétences digitales et d'être acteurs de la réalisation finale globale. En ce qui concerne l'évolution de leurs niveaux dans Minecraft, ils sont $75 \%$ à se qualifier de "Bon" ou « Expert » dans la maitrise du jeu en fin d'année scolaire, alors qu'ils étaient seulement $44 \%$ à s'identifier à ces catégories au début du semestre. 
Figure 3 : Auto-évaluation des élèves au début des séances Minecraft

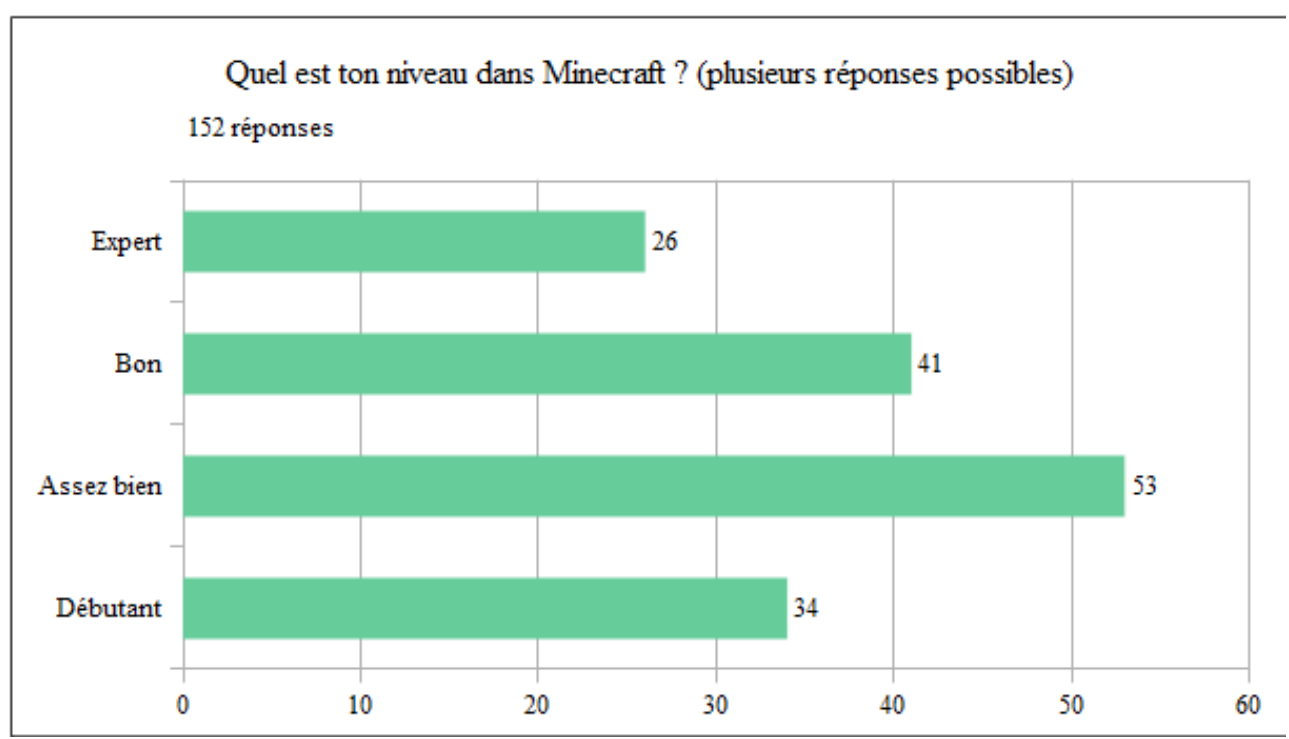

Figure 4 : Auto-évaluation des élèves à la fin des séances Minecraft

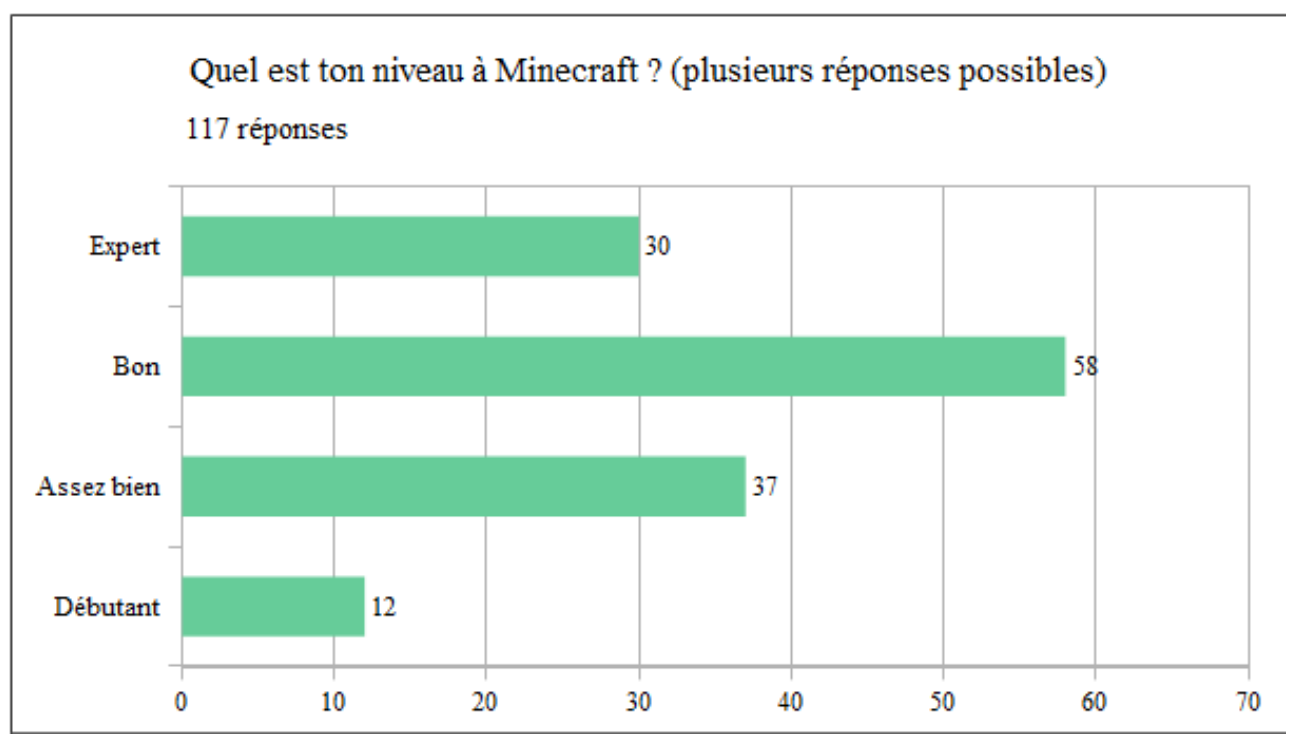

Ils considèrent l'expérience de ces séances comme une activité très sérieuse. Lors du bilan, plusieurs élèves insistent sur le fait que «ce qu'ils n'ont pas aimé dans les cours ", ce sont les attitudes négatives de leurs camarades qui soit détruisent les productions des autres (avec des blocs de $\mathrm{TNT}^{7}$ ), soit lancent des « potions d'invisibilité » qui provoquent la disparition de l'avatar du joueur. Ces perturbations, atteintes aux règles établies, démontrent aussi l'existence de ces dernières et qu'elles sont davantage mises en place par la situation d'enseignement et l'activité en groupe que par l'outil lui-même. Le bruit est également signalé comme élément perturbateur qui ne permet pas d'écouter les consignes de l'enseignant et limite l'attention. Leur motivation reste cependant forte. Ils sont majoritairement $(94 \%)$ prêts à participer à d'autres séances Minecraft l'année suivante sur d'autres projets de construction. 


\section{Conclusion}

31 Nous avons souhaité dans cette recherche inscrire la pratique scolaire de Minecraft dans une approche extensive des usages vidéoludiques des élèves. En effet, une meilleure connaissance des pratiques ludiques personnelles des élèves par les équipes enseignantes est un atout pour une utilisation pertinente du jeu dans les apprentissages scolaires. D'une part, parce qu'elle permet de comprendre ce que des élèves de 11-12 ans entendent par « jouer» dans un contexte de loisirs; et d'autre part, parce que cette connaissance aide à éclairer les choix des équipes pédagogiques quant aux titres de jeux choisis pour être employés en classe. Ainsi, si Minecraft.edu ne fait pas l'unanimité auprès des élèves de $5^{e}$ du collège observé, ce jeu reste une production populaire dont le titre "parle » aux élèves. Eux-mêmes ne mentionnent pas le suffixe «.edu» durant les entretiens, et les chercheuses ont tenté de ne pas encourager cette appellation afin de laisser le choix de la précision ou non aux élèves. La différence entre les deux versions est pourtant connue de tous, renforçant d'emblée le contraste entre les différents contextes de jeu avec le «vrai Minecraft » et le « Minecraft ici » (en classe).

32 L'impact positif d'un jeu sur les apprentissages est étroitement lié à la réflexion sur les conditions d'utilisation et les objectifs poursuivis. «Ce n'est pas seulement en raison de ses effets, de sa possible utilisation pédagogique, que le jeu revêt une importance et comporte une part essentielle de sérieux : c'est avant tout parce qu'il se présente ainsi au regard du joueur lui-même» (Henriot, 1989, p. 201). Non seulement le jeu ne peut se suffire à lui-même et doit faire partie d'un scénario pédagogique, mais il doit être inclus dans un dispositif contextuel qui permet de l'appréhender comme outil d'apprentissage, en prenant en compte les perceptions qu'ont les élèves du jeu en dehors de la salle de classe. Seule la médiation de l'enseignant peut donner du sens afin de permettre à l'élève de dépasser la simple expérience de jeu et de l'inscrire dans une véritable démarche d'apprentissage. Cette réflexion sur la médiation apparait indispensable si l'on souhaite vaincre les réticences des enseignants et développer les usages de cet outil.

Néanmoins, si les apports pédagogiques sont importants, la qualité de l'utilisation d'un outil tel que Minecraft.edu en éducation repose avant tout sur un équilibre entre contenu pédagogique et qualité ludique. Certes, ce titre a le mérite de rappeler le célèbre jeu vidéo, mais lui-même est difficilement catégorisable en tant que tel. La version « .edu ", qui est présentée comme un outil pédagogique, révèle à travers ses caractéristiques propres une différenciation forte entre pratiques de jeu en classe et pratiques de jeu hors de la classe. Alors que le « jeu » qui se déroule en contexte scolaire est ainsi défini par les équipes composées d'adultes, de nombreux élèves annoncent ne pas jouer. L'activité menée en classe avec Minecraft.edu varie donc entre "séance de jeu» et "séance de cours", tout comme le dispositif qui supporte cette activité oscille entre "jeu vidéo » et " outil pédagogique ». Les objectifs pédagogiques des enseignants semblent atteints. Les appropriations du dispositif par les élèves révèlent à la fois de « vrais » moments de jeu et de «vrais» apprentissages. Si la question de ce qu'est le «jeu» pour les élèves est souvent interrogée dans cette enquête, celle de ce qui est entendu par "apprentissage » est évoquée mais elle reste encore à explorer notamment au regard des systèmes d'évaluation en cours d'évolution. Il y a là matière à enrichir ces questionnements autour des liens entre jeu et apprentissages, mettant à nouveau la parole des élèves à contribution afin d'éclairer leur conception de ce que signifie « apprendre ». 


\section{BIBLIOGRAPHIE}

ALVAREZ, J., DJAOUTI, D. (2012), Introduction au Serious Game, $2^{\circ}$ édition, Paris, Questions théoriques.

ALVAREZ, J., DJAOUTI, D., RAMPNOUX, O. (2016), Apprendre avec les Serious Games ? Paris, Canopé Editions.

BERRY, V. (2011), « Jouer pour apprendre : est-ce bien sérieux ? Réflexions théoriques sur les relations entre jeu (vidéo) et apprentissage », Canadian Journal of Learning and Technology, $\mathrm{n}^{\circ} 37$, 2011.

BROUGERE, G. (1995), Jeu et éducation, Paris, L'Harmattan.

BROUGERE, G. (2002), « Jeu et loisir comme espaces d'apprentissages informels ». Education et sociétés, 10,2 , pp. 5-20.

COAVOUX S., RUFAT, S., TER MINASSIAN, H. (2014), « Jouer aux jeux vidéo en France : Géographie sociale d'une pratique culturelle ». L'Espace géographique, 43, 4, pp. 308-323.

DUMAS, P. (2004). « Nouveaux dispositifs pédagogiques et crise des systèmes éducatifs », Humanisme et entreprise. https://hal.archives-ouvertes.fr/sic_00000958/document

HENRIOT, J. (1989), Sous couleur de jouer, Paris, José Corti.

LAVIGNE, M. (2016), « Jeu et non jeu dans les serious games », Sciences du jeu n 5, https:// journals.openedition.org/sdj/648

NEBEL, S., SCHNEIDER, S., REY, G. D. (2016). « Mining learning and crafting scientific experiments: a literature review on the use of Minecraft in education and research ». Journal of Educational Technology \& Society, 19, 2, pp. 355-366.

OCTOBRE, S. (2004), Les loisirs culturels des 6-14 ans. Paris, La Documentation française, Ministère de la culture et de la communication, Département des études et de la prospective.

TREMEL, L. (2007). « Jeux, éducation et socialisation politique : contribution au rappel de la permanence d'un processus ». Géographie, économie, société, 9, 1, pp. 83-99.

\section{NOTES}

1. Les jeux de type "bac à sable » ou sandbox en anglais sont des jeux qui ne proposent pas d'objectifs prédéterminés aux joueurs, ces derniers étant ainsi libres de se créer leurs propres objectifs de jeu.

2. Minecraft classique est utilisable selon quatre modes possibles : créatif, survie, aventure ou hardcore. Ce dernier mode de jeu, dérivant du mode survie, implique l'impossibilité de réapparaître après la mort si ce n'est en mode spectateur.

3. https://education.minecraft.net/

4. Enquête PELLEAS, OFDT-CJC Pierre-Nicole, Croix-Rouge française, 2014.

5. Les diffusions en mode continu ou stream en anglais sont des parties de jeu vidéo enregistrées par d'autres joueurs, parfois diffusées en livestream, c'est-à-dire en direct, sur des plateformes telles que Twitch.fr ou Youtube. Ces formats rencontrent un succès grandissant. 
6. Le mode «survie » est un mode de jeu dans lequel les joueurs doivent collecter des ressources, bâtir des structures, combattre des monstres, gérer leur faim et explorer le monde pour survivre et prospérer.

7. Le TNT (Trinitrotoluène) est un bloc qui une fois activé finit par exploser, pouvant ainsi infliger de lourds dégâts.

\section{RÉSUMÉS}

Ce texte présente une étude menée à partir de l'expérience du jeu Minecraft.edu auprès de collégiens dans un cadre interdisciplinaire. Notre réflexion s'appuie sur une enquête auprès des participants du projet et des observations directes de séances pédagogiques afin de croiser données qualitatives et quantitatives, informations déclaratives et observées. Cette contribution se propose d'éclairer les pratiques vidéoludiques des jeunes élèves dans le contexte familial et éducatif. Dans une perspective culturelle, nous réfléchissons au rapport entre l'apprentissage et la jouabilité (gameplay) dans un jeu sérieux.

This article presents a study about the experience of MinecraftEdu, during class with middle school students. This experience is lead through an interdisciplinary framework. Our thinking is based on an inquiry with participants from the project and observations during classes. The aim is to collect and juxtapose both qualitative and quantitative data, as well as declarative and observed data. This contribution proposes to highlight young people video game activities in a familial and educative context. Through a cultural perspective, this inquiry interrogates the links between learning and play in a serious game.

\section{INDEX}

Mots-clés : jeu vidéo, enseignement, serious games, Minecraft, intelligence collective

Keywords : video game, education, serious games, Minecraft, collective intelligence

\section{AUTEURS}

\section{LETICIA ANDLAUER}

Université de Lille

FLORENCE THIAULT

Université de Lille

\section{LAURE BOLKA-TABARY}

Université de Lille 